\title{
Plasmonics in Future Information and Energy Generation Technologies
}

\author{
Harry A. Atwater \\ Thomas J. Watson Laboratories of Applied Physics and Kavli Nanoscience Institute, \\ California Institute of Technology, Pasadena, CA 91125 USA haa@caltech.edu
}

\begin{abstract}
Plasmonics has provided researchers new means to control optical dispersion and light localization at nanoscale dimensions. Plasmonic design approaches are yielding building blocks for chip-based optical components as well as optical metamaterials and light trapping structures for improved efficiency in thin solar cells.
\end{abstract}

\section{Introduction}

Since 2003, there has been an explosive growth of scientific interest in the role of surface plasmons in optical phenomena. Surface plasmons are charge oscillations of free electrons near a metal surface. In threedimensional geometries, such as single metal nanoparticles or arrays of coupled nanoparticles, surface plasmons lead to resonant scattering and absorption due to localized resonances. At a planar metal/dielectric interface, surface plasmons cause propagating solutions of Maxewell's equations called surface plasmon polaritons (SPPs), that are essentially two-dimensional waves, with the electromagnetic field well confined to the interface, as depicted in Fig. 1. The resonance frequency of both these localized and propagating plasmons can be tuned over the visible and near infrared spectral range by using metals such as $\mathrm{Ag}, \mathrm{Au}, \mathrm{Cu}$ and $\mathrm{Al}$ in combination with dielectrics such as $\mathrm{SiO}_{2}$, $\mathrm{Si}_{3} \mathrm{~N}_{4}$, or semiconductors such as $\mathrm{Si}, \mathrm{GaAs}$, and $\mathrm{CdTe}$. Near the plasmon resonance frequency, the excited localized or propagating modes access a large range of wavevectors and the electromagnetic fields are confined to dimensions much smaller than the wavelength.

Surface plasmon research developed initially in the 1980s concurrently with rapid progress in surface science, in large part due to intensive work on surface-enhanced Raman scattering near small metal particles. However in the last few years, the young field of plasmonics has been rapidly expanding as a new materials and devices research area. This is a result of the large array of nanoscale fabrication and nanophotonics characterization tools that have become quite widely available, and also the availability of powerful electromagnetic simulation methods that are critical to understanding and usefully harnessing plasmon excitations. Studies illustrating the coupling of plasmons to optical emitters [1, 2] and phasechange media to achieve active plasmonics [3], plasmon focusing $[4,5]$, nanoscale wave guiding, [6-9] nanoscale antennas [10], plasmonic integrated circuits [11,12], plasmonic lasers [13-14], and surface plasmon-enhanced LED characteristics [15] have been reported. The attention to plasmonic materials in photonics was also bolstered by the observation in 1998 of extraordinarily high transmission through small arrays of holes in metal films.[16] The field of plasmonics received yet another boost with predictions of 'perfect' lens or superlens behavior,[17] using materials with negative refractive index. The design and fabrication of such optical "metamaterials" of which the building blocks are composed of plasmonic nanostructures with features much smaller than the wavelength has become an active field of research [18-20].

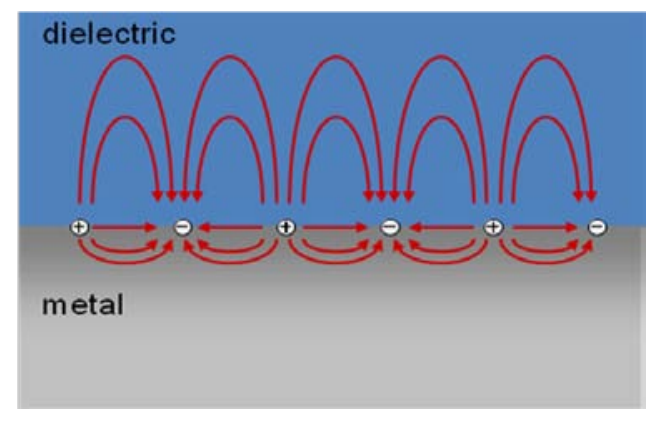

Fig. 1: Surface plasmons are longitudinal charge density fluctuations at the interface between a conductor and a dielectric. The periodically-polarized charge density couples to the electromagnetic field to generate a guided wave mode localized to the metal/dielectric interface. 


\section{Nanoscale Light Sources, Waveguides and Modulators for Intrachip-Based Photonics}

Since the inception of plasmonics research there has been great interest in development of chip-based plasmonic networks suitable for intrachip and interchip optical communication. Initial work in the field focused on the properties of surface plasmon polariton (SPP) waveguides and researchers envisioned that all-metal photonic interconnects might have potential for on-chip photonics. However it was found that the trade-off between light localization and waveguide propagation loss favoured either SPP waveguides with large mode volume (similar to modes in single-mode optical fibres) and low propagation losses or highly-localized $(<100 \mathrm{~nm})$ modes with propagation distances in the tens of microns. This realization led to a new hybrid plasmonic/silicon photonic network approach, depicted in Fig. 2, utilizing silicon-oninsulator waveguides for across-chip

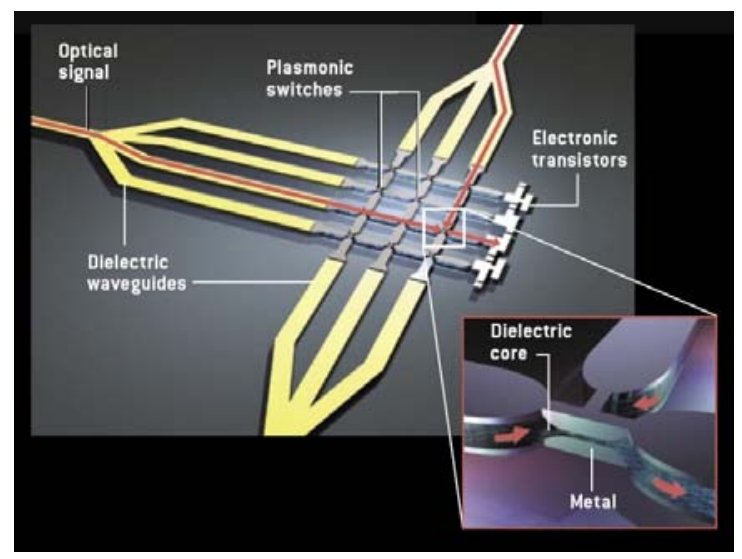

Fig. 2: Schematic of chip-based hybrid silicon/plasmonic network. Across-chip waveguides are realized in silicon-on-insulator technology, whereas nanoscale waveguides and modulators employ plasmonic designs.

interconnects and plasmonic structures for ultracompact active devices (modulators, lasers, LEDs, detectors) as well as nanoscale waveguides.

Metal-dielectric plasmon waveguides can serve as active switching elements when the dielectric refractive index can be actively modulated. In 2007, we demonstrated plasmonic all-optical modulation in which the complex mode refractive index seen by a surface plasmon polariton at infrared free-space wavelength $(\lambda=1.42 \mu \mathrm{m})$ is modulated via interband excitation of the dielectric medium at visible frequencies $(\lambda=0.514 \mu \mathrm{m})$ [21]. We also recently demonstrated electro-optic refractive index modulation in metal-dielectric-metal plasmon waveguides using low-voltage electrooptic modulation of perovskite oxide dielectric layers [22]. Most interesting for silicon-based photonic integrated circuits and silicon electronics is the realization of fully CMOScompatible active switching devices. To this end, we also demonstrated in 2009 a "plasMOStor"[23,24], which is an active Si field effect device that modulates the complex refractive mode index for plasmonic modes propagating in the channel of a silicon MOS field effect device. The plasMOStor demonstrated on/off switching ratios of $11.2 \mathrm{~dB}$ and device capacitance-limited switching speeds and potential for ultralow power dissipation.

\section{Plasmonic for Improved Photovoltaics}

Until recently, little systematic, comprehensive thought has been given to the question of how the novel concepts of plasmon excitation and light localization described above might be exploited to advantage in high efficiency photovoltaics. Conventionally, photovoltaic absorbers must be optically 'thick' to enable nearly complete light absorption and photocarrier current collection. For example, the $600-1100 \mathrm{~nm}$ spectral range of the standard AM1.5 solar spectrum is poorly absorbed upon a single pass through a thin $(<10 \mu \mathrm{m}) \mathrm{Si}$ film. This is the reason that conventional wafer-based crystalline Si solar cells have a thickness of typically $300 \mu \mathrm{m}$. An additional requirement for high efficiency solar cells is that they must have minority carrier diffusion lengths several times the material thickness in order for all photocarriers to be collected, a requirement which is best met for thin cells. Thus solar cell design and material synthesis considerations are strongly dictated by these opposing optical absorption thickness and carrier collection length requirements.

Plasmonic structures can offer the possibility of reducing the physical thickness of the photovoltaic absorber layers while keeping their 'optical thickness' constant, in at least two ways[25]:

1) Metallic nanoparticles can be used as subwavelength scattering elements located at the front or back of a thick (relative to the wavelength) solar cell to scatter freely propagating plane waves from the sun into large scattering angles in the cell, thereby increasing the optical path length with the structure.

2) A corrugated metallic film on the back or front surface of a thin (wavelength-scale) photovoltaic absorber film can couple sunlight into guided modes of an absorbing semiconductor; whereupon the light is converted to photocarriers in the semiconductor.

Thus the efficiency and cost effectiveness of photovoltaic cells can both be increased by reduction of the active semiconductor absorber 
layer thickness and ability to fabricate ultrathin absorber layers opens up new possibilities for solar cell device design. Enhanced photocurrent and efficiency has been demonstrated for thin a-Si[26] and GaAs solar cells[27]. Modal analysis in full wave simulation allows us to determine the fraction of power absorbed by the solar cell to be determined for both dielectric and plasmonic modes[28]. Interestingly, it also appears possible to utilize nanoscale plasmonic light trapping structures to significantly exceed the previously anticipated limits to light trapping in photovoltaics[29].

\section{References}

1 P. Andrew, and W.L. Barnes, Science 306, 1002 (2004).

2 H. Mertens, J.S. Biteen, H.A. Atwater, and A. Polman, Nano Lett. 6, 2622 (2006)

3 A.V. Krasavin, and N.I. Zheludev, Appl. Phys. Lett., 84, pp 1416-1418 (2004).

4 M. I. Stockman, Phys. Rev. Lett. 93, 137404 (2004).

5 E. Verhagen, A. Polman and L. Kuipers, Optics Express 16, 45 (2008).

6 M. Quinten, A. Leitner, J.R. Krenn, and F.R. Aussenegg, Opt. Lett. 23, 1331 (1998).

7 A.F. Koenderink and A. Polman, Phys. Rev. B 74, 033402 (2006)

8 S.A. Maier, P.G. Kik, H.A. Atwater, S. Meltzer, E. Harel, B.E. Koel, and A.A.G. Requicha, Nature Mater. 2, 229 (2003).

9 S.A. Maier and H.A. Atwater, J. Appl. Phys. 98 Article Number: 011101 (2005).

10 D.M. O'Carroll, C.E. Hofmann, H.A. Atwater, Advanced Materials 22 pp 1223-+ (2010).

11 H. Ditlbacher, J.R. Krenn , G. Schider, A. Leitner, and F.R. Aussenegg, Appl. Phys. Lett. 81, 1762 (2002).

12 S. I. Bozhevolnyi, V. S. Volkov, E. Devaux, J.-Y. Laluet, and T. W. Ebbesen, Nature 440, 508 (2006).

13 R. Colombelli, K. Srinivasan, M. Troccoli, O. Painter, C.F. Gmachl, D.M. Tennant, A.M. Sergent, D.L. Sivco, A.Y. Cho, and F.
Capasso, Science 302, 1374 (2003); M.T. Hill et al., Nature Photonics 1, 589 (2007)

14 R.F. Oulton, V.J. Sorger, T. Zentgraf, R.M. Ma, C. Gladden, G. Bartal and X. Zhang, Nature 461 pp 629-632 (2009).

15 K. Okamoto, I. Niki, A. Shvartser, Y. Narukawa, T. Mukai, A. Scherer, Nature Materials 3, 601 (2004).

16 T.W. Ebbesen, H.J. Lezec, H.F. Ghaemi, T. Thio, and P.A. Wolff, Nature 391, 667 (1998).

17 J.B. Pendry, Phys. Rev. Lett. 85, 3966 (2000).

18 R.A. Shelby, D.R. Smith, and S. Schultz, Science 292, 77 (2001).

19 S. Linden, C. Enkrich, M. Wegener, J. Zhou, T. Koschny, and C.M. Soukoulis, Science 306, 1351 (2004).

20 N. Fang, H. Lee, C. Sun, and X. Zhang, Science 308, 534 (2005).

21D.Pacifici, H.J. Lezec, and H.A. Atwater, Nature Photonics 1 pp 402-406 (2007).

22 M.J. Dicken, L.A. Sweatlock, D. Pacifici, H.J Lezec, K. Bhattacharya and H.A. Atwater, Nano Letters 8 pp 4048-4052 (2008).

23 J.A. Dionne, K. Diest, L.A. Sweatlock, H.A. Atwater, Nano Letters 9, pp 897-902 (2009).

24 J.A. Dionne, L.A. Sweatlock, M.T. Sheldon, A.P. Alivisatos and H.A. Atwater, IEEE J. Selected Topics In Quantum Electronics, 16, pp 295-306 (2010)

25 H.A. Atwater, and A. Polman, Nature Materials 9 pp 205-213 (2010).

26 VE Ferry, MA Verschuuren, HB Li, R.E.I. Schropp, H.A. Atwater and A. Polman, Appl. Phys. Lett., 95 Article Number: 183503 (2009).

27 K. Nakayama, K.Tanabe, and H.A. Atwater, Appl. Phys. Lett., 93 Article Number: 121904 (2008).

28 V.E. Ferry, L.A. Sweatlock, D. Pacifici, and H.A. Atwater, Nano Letters 8 pp 43914397(2008)

29 P.N. Saeta, V.E. Ferry, D. Pacifici, J.N. Munday and H.A. Atwater, Optics Express 17 pp 20975-20990 (2009). 